\title{
ChemComm
}

\section{A non-aqueous procedure to synthesize amino group bearing nanostructured organic-inorganic hybrid materials $\dagger$}

50, 9753

Received 14th May 2014

Accepted 7th July 2014

DOI: $10.1039 / c 4 c c 03640 a$

www.rsc.org/chemcomm

Amino-functionalized organic-inorganic hybrid materials with a narrow distributed nanostructure of 2-4 $\mathrm{nm}$ in size were obtained by means of a template-free and non-aqueous procedure. Simultaneous twin polymerization of novel amino group containing twin monomers with 2,2'-spirobi[4H-1,3,2-benzodioxasiline] has been applied for this purpose. The amino groups of the organic-inorganic hybrid material are useful for post derivatization.

Organic-inorganic hybrid materials, which contain amino groups, are of great importance for a variety of applications such as supports for catalysts, adsorption of metal ions, or as key precursors for post derivatization. ${ }^{1,2}$ It is an important task to obtain nanostructured materials with accessible amino groups due to their great potential for post-functionalization reactions with electrophilic reagents. ${ }^{3-5}$ Amino functionalized silica materials have been successfully used for $\mathrm{CO}_{2},{ }^{6}$ water $^{7,8}$ or dye absorption ${ }^{7}$ and catalysis of Knoevenagel condensation reactions ${ }^{9,10}$ and the synthesis of nitrostyrenes. ${ }^{11}$ Furthermore, primary and secondary amino groups can be modified in many ways to introduce carboxyl groups from cyclic anhydrides or imine groups from aldehydes.

These materials can be synthesized by different strategies which use amino-functionalized reagents. ${ }^{1,2,8,12,13}$ Suitable reagents are 3-aminopropylalkoxysilanes or amino group bearing water soluble polymers such as polyvinylamine. ${ }^{13}$ The synthetic procedure employing these reagents is based on water as a co-reagent or a solvent. Therefore, co-reagents which contain hydrolytically sensitive groups such as imines or isocyanates cannot by applied when water based procedures are used. To avoid these peculiarities, a synthetic method is needed which does not require water as a solvent or a reagent.

\footnotetext{
${ }^{a}$ Department of Polymer Chemistry, Institute of Chemistry,

Technische Universität Chemnitz, Strasse der Nationen 62, D-09111 Chemnitz, Germany.E-mail: stefan.spange@chemie.tu-chemnitz.de

${ }^{b}$ BASF SE, Carl-Bosch Straße 38, D-67056 Ludwigshafen, Germany

$\dagger$ Electronic supplementary information (ESI) available: Experimental information, CP-MAS-NMR-spectra, IR-spectra, DSC measurements, TEM images and ${ }^{1} \mathrm{H}$-NMR-spectra. See DOI: 10.1039/c4cc03640a
}

Twin-polymerization can be carried out either in organic solvents or even in the melt without the use of any solvent. ${ }^{14,15}$ This advantage overcomes all problems resulting from water chemistry. Furthermore, amino groups undergo acid-base reactions with water, and ammonium ions are formed which can have a negative effect on post-reactions of amino groups.

In this communication a specific application of twinpolymerization is presented which uses a non-aqueous procedure involving various amino group bearing twin monomers to produce nanostructured organic-inorganic hybrid materials related to amino group containing sol-gel materials.

During the last six years the so called twin polymerization has been developed to fabricate organic-inorganic hybrid materials by solely one procedure. ${ }^{14-18}$ The principle of twin-polymerization is based on specific twin monomers (TMs), which contain two covalently bonded polymerizable monomeric fragments, i.e. A and B for each polymer. The formation of the two polymers from the twin monomers during polymerization is mechanistically coupled. ${ }^{17}$ Thus, polymer $-(\mathrm{A})_{n}-$ can only be formed when polymer $-(\mathrm{B})_{n}-$ is also formed. This is a crucial difference in the polymerization behavior from that of hetero-bifunctional monomers whose two different polymer strands are mechanistically independent of each other during the polymerization procedure. ${ }^{4,5,13}$ Simultaneous twin polymerization (STP) of two different twin monomers in one process can yield up to four different polymers (see also Fig. S1 ESI $\dagger$ ). ${ }^{18}$

A benefit of this type of STP is that the formation of polymer -(A) $n^{-}$mediates the covalent connection of fragments $\mathrm{B}$ and $\mathrm{C}$ during polymerization within the organic-inorganic hybrid material.

The synthetic procedure for the amino group containing TM (2-5) starts from salicylic alcohol and 3-amino-n-propyldimethoxymethylsilanes. The transesterification reaction was catalyzed by tetra- $n$-butylammonium fluoride (TBAF) (see Fig. 2). Details of the synthetic procedures are given in the ESI. $\dagger$

Objective of this basic study is the synthetic feasibility of new types of TMs and to study their polymerization by STP to produce hybrid materials bearing accessible amino groups. The STP of 2,2'-spirobi[4H-1,3,2-benzodioxasiline] (1) with amino group functionalized twin monomers has been studied. 


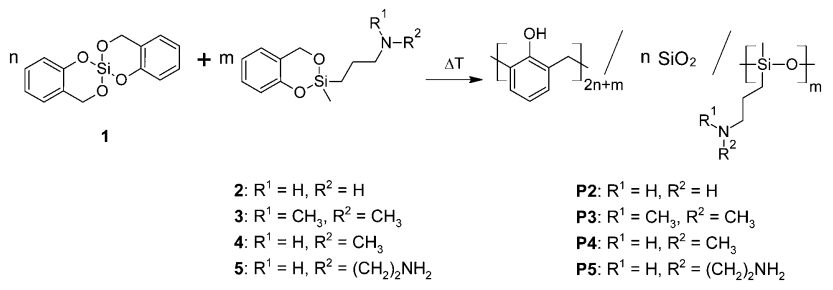

Fig. 1 Procedure for thermally induced STP of twin monomer $\mathbf{1}$ with 2, 3, 4 and 5 , respectively, and appellation of the resulting hybrid materials.

Pure 1 undergoes twin polymerization by either an acid- or a base-catalyzed reaction in melt or in solution. ${ }^{19,20}$ However, it is also possible to polymerize 1 without the use of any catalyst at $230{ }^{\circ} \mathrm{C}^{19}$

Advantageously, the amino functionalized monomers (2-5) used for the STP can serve as both a component and a basic catalyst. This is an elegant way of synthesis, because impurities resulting from the use of additional catalysts can be avoided. Thus, monomer 1 was simultaneously polymerized with $2,3,4$ or 5 at a much lower temperature $\left(120^{\circ} \mathrm{C}\right)$ using a stoichiometric ratio of $1: 1$.

According to the conceptual idea of STP, an organic-inorganic hybrid material is composed of phenolic resin, silica and oligo(3-amino- $n$-propyl)methylsiloxane (OAMS) (Fig. 1).

It is apparent from silicon chemistry that silica and OAMS moieties can undergo $\mathrm{Si}-\mathrm{O}-\mathrm{Si}$ bond formation to form a class II hybrid structure among the phenolic resin/ $/ \mathrm{SiO}_{2} / \mathrm{OAMS}$ hybrid compound. ${ }^{21-23}$ This feature can be readily evidenced by solid state ${ }^{29} \mathrm{Si}-$ and ${ }^{13} \mathrm{C}-\mathrm{NMR}$ spectroscopy shown in Fig. 2 and the ESI. $\dagger$

Altogether, the ${ }^{29} \mathrm{Si}-\mathrm{CP}-\mathrm{MAS}-\mathrm{NMR}$ spectra of hybrid materials (P2-P5) show the expected Q- and D-signals originating from monomer 1 and the accordant amino functionalized monomers (2-5). In each case $\mathrm{Q}_{4}(-110 \mathrm{ppm})$ is the most intense signal in the silica region of the spectrum, although the cross polarization technique used overrates $\mathrm{Q}_{2}(-90 \mathrm{ppm})$ and $\mathrm{Q}_{3}(-100 \mathrm{ppm})$ signals due to polarization transfer from ${ }^{1} \mathrm{H}$ to ${ }^{29} \mathrm{Si}$. This result indicates that the silica network is highly condensed. The same applies to the OAMS part, as no signals from the monomer are detectable. Instead, a rather broad signal at $-\mathbf{1 7 . 6} \mathrm{ppm}$ is found,
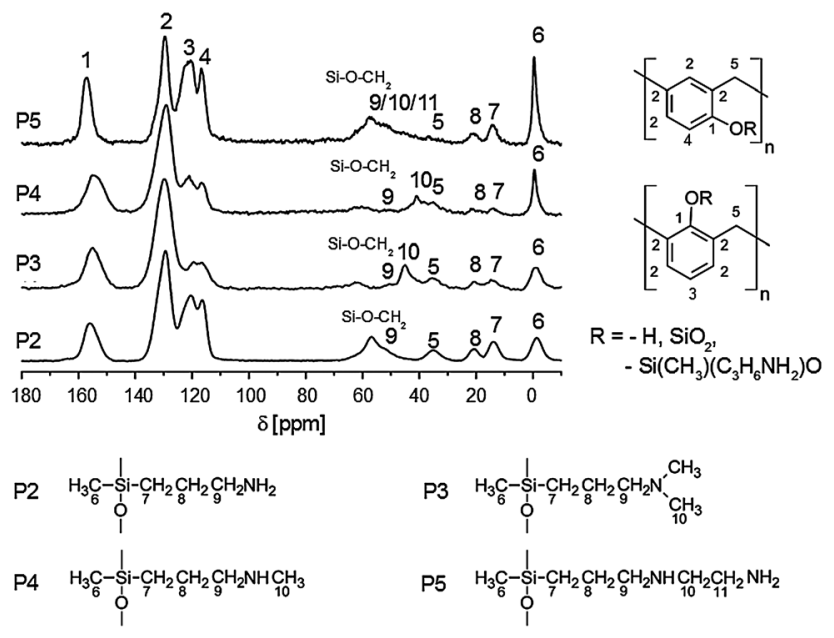

Fig. 2 Solid state ${ }^{13} \mathrm{C}-\left\{{ }^{1} \mathrm{H}\right\}-\mathrm{CP}-\mathrm{MAS}-\mathrm{NMR}$ spectra of samples P2-P5. which can be assigned as $\mathrm{D}(\mathrm{Q}) \cdot{ }^{24}$ Parts of OAMS are covalently bonded to silica and form a copolymer, related to Co-STP as shown in Fig. 1, ESI. $\dagger$ The broad line widths of the D(Q)-signals are typical for a high dispersion of chemical shifts and a reduced flexibility of the OAMS moiety. The formation of phenolic resin and of OAMS can be clearly evidenced by means of ${ }^{13} \mathrm{C}-\mathrm{CP}-\mathrm{MAS}-\mathrm{NMR}$ spectroscopy. The signal-structure assignments are depicted in Fig. 2. The formation of $o, o^{\prime}$ - and $o, p^{\prime}$-linked phenolic resin structures could be evidenced by the signal pattern in the aromatic carbon region (signals 3 and 4, Fig. 2) and a broad signal of the bridging methylene groups (signal 5). An intense signal of methyl groups of OAMS arises at $0 \mathrm{ppm}$. The other carbon atoms of the alkyl chains of OAMS are found in the expected regions (10-50 ppm). The weak signal at $62 \mathrm{ppm}$ originates from unreacted $\mathrm{Si}-\mathrm{O}-\mathrm{CH}_{2}$ groups of the monomers (1: $66.3 \mathrm{ppm} ; 2$, 3, 4 and 5: $\sim 63 \mathrm{ppm})$ or benzyl alcohol $\left(-\mathrm{CH}_{2}-\mathrm{OH}: 64.7 \mathrm{ppm}\right)$. This is in agreement with the results of the ${ }^{29} \mathrm{Si}-\mathrm{NMR}\left(\mathrm{Q}_{3}\right)$, and post-reactions above $120{ }^{\circ} \mathrm{C}$ were detected by differential scanning calorimetry (DSC) measurements (Fig. S5 ESI $\dagger$ ). Soxhlet extraction with dichloromethane (DCM) showed extractable fractions which are attributed to low molecular weight products (see ESI $\dagger$ ). A high degree of polymerization in the case of step growth polymerizations is only achieved with nearly complete conversion of functional groups. Thus, composites of monomers $\mathbf{1}$ and $\mathbf{2}$ were synthesized at higher temperatures $\left(180{ }^{\circ} \mathrm{C}\right.$; monomer 2 solely polymerizes at $\left.220{ }^{\circ} \mathrm{C}\right)$ and in different ratios to P2_1 (1:2 = 95: 05), P2_2 (85:15), P2_3 (50:50), P2_4 (15:85) and P2_5 (0:100) (experimental details are given in the ESI $\dagger$ ). The higher polymerization temperature reduces the extractable content of the resulting hybrid material significantly from $43 \mathrm{wt} \%$ (P2) to $6 \mathrm{wt} \%$ (P2_3), see ESI. $\dagger$ DSC-measurements show no more post-reactions upon thermal treatment of the materials (see $\mathrm{ESI} \dagger$ ). The molecular structure monitored by means of ${ }^{13} \mathrm{C}$ - and ${ }^{29} \mathrm{Si}-\mathrm{CP}-\mathrm{MAS}-\mathrm{NMR}$ spectroscopy is not altered by the increased temperature (P2 Fig. S2 vs. P2_3 Fig. S3 ESI $\dagger$ ).

The solid state NMR spectra again evidence the formation of the phenolic resin, silica and OAMS (Fig. S3 ESI $\dagger$ ). The different monomer ratios are reflected in the NMR spectra and the signal intensities of phenolic resin/OAMS change accordingly. The ${ }^{13} \mathrm{C}$-NMR spectra show no differences in the chemical shifts. An increasing content of monomer 2 also leads to more intense D-signals as shown in the ${ }^{29}$ Si-NMR spectra (Fig. 3). A low ratio of monomer 2 results in $\mathrm{D}(\mathrm{Q})$ species. The corresponding signal arises

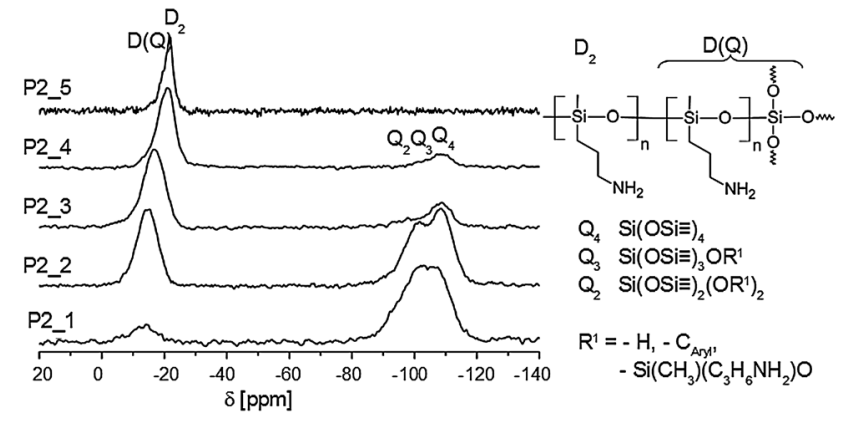

Fig. 3 Solid state ${ }^{29} \mathrm{Si}-\left\{{ }^{1} \mathrm{H}\right\}-\mathrm{CP}-\mathrm{MAS}-\mathrm{NMR}$ spectra of samples P2_1-P2_5. 
at $-17 \mathrm{ppm}$. Because of the formation of the silica-OAMS copolymer, the extractable content of sample P2_1 (0.5 wt\%) is low. Longer chains (or rings) of OAMS are formed upon increasing content of monomer 2 . The signal is shifted to a higher field $(-21 \mathrm{ppm})$ related to $\mathrm{D}_{2}$ structures. ${ }^{24}$ Also a larger quantity of OAMS could be extracted (P2_4: $31.2 \mathrm{wt} \%$ ) because less covalent bonds were formed between the silica network and OAMS. Due to the absence of monomer 1 the material P2_5 only shows D- without any Q signals. The intensities of the Q signals of P2_1 to P2_4 are affected by the monomer ratio used. A reduction of monomer 2 leads to increased $Q_{3}$ signals because the concentration of the basic catalyst (2) is decreased.

High angle annular dark field (HAADF) scanning transmission electronmicroscopy (STEM) images of selected hybrid materials show domain sizes of 2-4 nm (Fig. 4a). This is much lower than comparable phenolic resin/silica composites synthesized by a solgel process. ${ }^{25,26}$ The materials are transparent and no macroscopic agglomeration could be observed in all cases (Fig. 4b and ESI $\dagger$ ).

Thermogravimetric analysis (TGA) of the organic-inorganic hybrid materials shows a slight weight loss of $2.11 \mathrm{wt} \%$ (P2_1) to 8.66 wt\% (P2_5) between 30 and $200{ }^{\circ} \mathrm{C}$. Further decomposition of the hybrid material is comparable to that of aminopropylmodified hybrid materials known from the literature. ${ }^{12}$

In order to check the accessibility of the amino groups in the hybrid materials after STP, the reaction with different aldehydes was studied (Fig. 5). After washing and drying procedures, the formation of the Schiff base was examined by infrared- (IR) and ultraviolet-visible (UV/Vis) spectroscopy. The treated materials show additional IR-bands at 2230, 1643 and $696 \mathrm{~cm}^{-1}$ belonging to $\mathrm{C} \equiv \mathrm{N}$ and $\mathrm{C}=\mathrm{N}$ vibrations and at $1346 \mathrm{~cm}^{-1}$ assigned to the $\mathrm{N}-\mathrm{O}$ vibration of the nitro group (see ESI $\dagger$ ). The conversion of the amino groups within the hybrid material was $14.4-66.7 \%$ determined by quantitative elemental analysis of the $\mathrm{N}$-content with respect to $100 \%$ theoretical conversion (see ESI $\dagger$ ).

The functionalized hybrid materials SB1-3 obtained have yellow to orange color shades. UV/Vis absorption spectra of the untreated composite P2_3 and SB1-3 have been recorded by means of diffuse reflectance UV/Vis spectroscopy. The UV/Vis absorption bands are non-symmetric and show several UV/Vis absorption maxima. A bathochromic shift of the UV/Vis absorption band can be observed for Schiff bases SB1-3 in comparison to the untreated composite material P2_3 $\left(\lambda_{\max }=294 \mathrm{~nm}\right)$ $\left(\Delta \tilde{v}\left(\mathbf{P 2} \_3 \rightarrow\right.\right.$ SB3 $\left.)=2268 \mathrm{~cm}^{-1}\right)$, which confirms the accessibility of the amino groups and the formation of Schiff bases (Fig. 6).

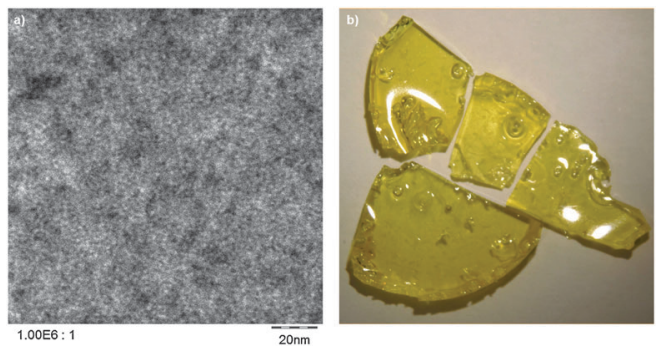

Fig. 4 (a) HAADF-STEM image of the composite material P2 showing domains of 2-4 $\mathrm{nm}$ in size. (b) Macroscopic picture of the transparent, monolithic composite material.

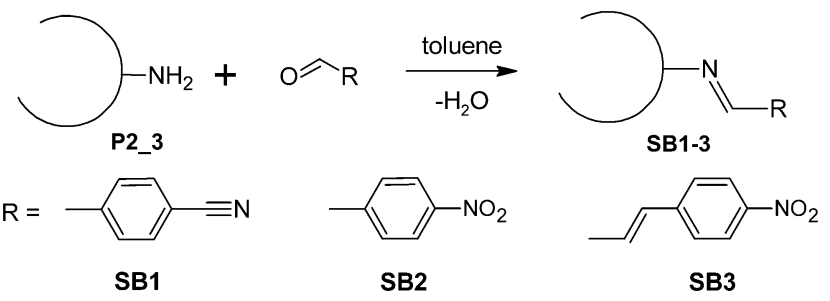

Fig. 5 Reaction of surface amino groups with $p$-cyanobenzaldehyde, $p$-nitrobenzaldehyde and $p$-nitro-cinnamaldehyde.

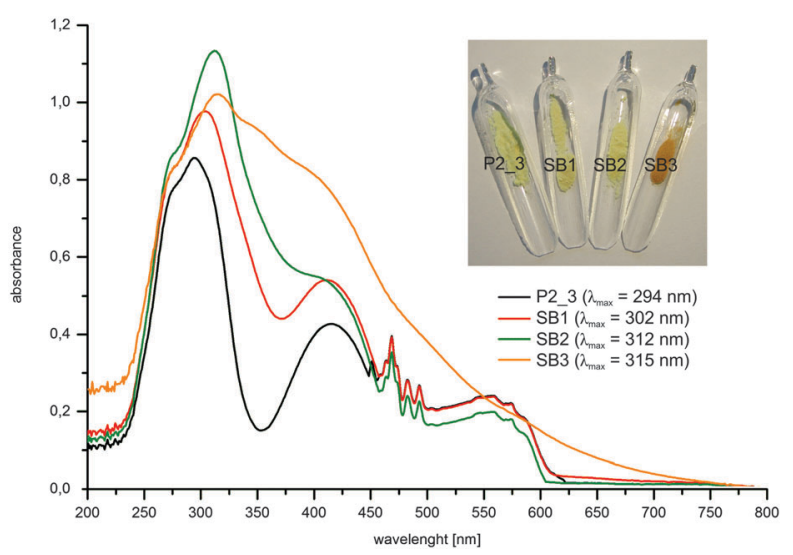

Fig. 6 UV/Vis absorption spectra of the untreated composite material P2_3 and SB1-SB3.

In addition, the UV/Vis absorption maximum of the Schiff bases shifts bathochromically from $302 \mathrm{~nm}$ (SB1) to $312 \mathrm{~nm}$ (SB2) and $315 \mathrm{~nm}$ (SB3). This is caused by an increase in the strength of the push-pull $\pi$-electron system with the increasing accepting capacity of the corresponding substituent $\left(\mathrm{CN} \rightarrow \mathrm{NO}_{2}\right)$.

We can conclude that monolithic nanostructured (phenolic resin/aminosilane/ $\mathrm{SiO}_{2}$ ) organic-inorganic hybrid materials obtained from 1 (2,2'-spirobi[4H-1,3,2-benzodioxazoiline]) and various N-substituted 2-(3-amino- $n$-propyl)-2-methyl-4H-1,3,2benzodioxazoiline derivatives (2-5) can be produced in the melt by self-induced simultaneous twin polymerization at $180{ }^{\circ} \mathrm{C}$. The molecular structures of the organic-inorganic hybrid materials have been evidenced by solid state ${ }^{13} \mathrm{C}$ - and ${ }^{29} \mathrm{Si}-\mathrm{NMR}$ spectroscopy. Intactness of the primary amino groups has been proved by reactions with aldehydes to achieve azomethine moieties (SB1-3), which can be detected readily by UV/Vis spectroscopy.

Application of this specific type of twin monomer combination in heat-induced coating, particle fabrication or other purposes will be demonstrated soon.

This work was performed within the Federal Cluster of Excellence EXC 1075 "MERGE Technologies for Multifunctional Lightweight Structures" and DFG SP 392/34-1, supported by the German Research Foundation (DFG). Financial support is gratefully acknowledged.

\section{Notes and references}

1 F. Hoffmann, M. Cornelius, J. Morell and M. Fröba, Angew. Chem., 2006, 118, 3290-3328. 
2 J. E. Lofgreen and G. A. Ozin, Chem. Soc. Rev., 2014, 43, 911-933.

3 L. Ni, N. Moreau, A. Chemtob and C. Croutxé-Barghorn, J. Sol-Gel Sci. Technol., 2012, 64, 500-509.

4 M. Sakeye and J.-H. Smått, Langmuir, 2012, 28, 16941-16950.

5 R. Voss, A. Thomas, M. Antonietti and G. A. Ozin, J. Mater. Chem., 2005, 15, 4010-4014.

6 S. Araki, H. Doi, Y. Sano, S. Tanaka and Y. Miyake, J. Colloid Interface Sci., 2009, 339, 382-389.

7 Z. Wu, H. Xiang, T. Kim, M.-S. Chun and K. Lee, J. Colloid Interface Sci., 2006, 304, 119-124.

8 G. G. Paradis, R. Kreiter, M. M. A. van Tuel, A. Nijmeijer and J. F. Vente, J. Mater. Chem., 2012, 22, 7258-7264.

9 D. J. Macquarrie and D. B. Jackson, Chem. Commun., 1997, 1781-1782.

10 B. M. Choudary, M. L. Kantam, P. Sreekanth, T. Bandopadhyay, F. Figueras and A. Tuel, J. Mol. Catal. A: Chem., 1999, 142, 361-365.

11 G. Sartori, J. Catal., 2004, 222, 410-418.

12 R. Brambilla, J. Poisson, C. Radtke, M. S. L. Miranda, M. B. Cardoso, I. S. Butler and J. H. Z. dos Santos, J. Sol-Gel Sci. Technol., 2011, 59, 135-144.

13 I. Voigt, F. Simon, K. Estel and S. Spange, Langmuir, 2001, 17, 3080-3086.

14 S. Grund, P. Kempe, G. Baumann, A. Seifert and S. Spange, Angew. Chem., Int. Ed., 2007, 46, 628-632.

15 S. Spange and S. Grund, Adv. Mater., 2009, 21, 2111-2116.
16 P. Kempe, T. Löschner, A. A. Auer, A. Seifert, G. Cox and S. Spange, Chem. - Eur. J., 2014, 20, 8040-8053.

17 A. A. Auer, A. Richter, A. V. Berezkin, D. V. Guseva and S. Spange, Macromol. Theory Simul., 2012, 21, 615-628.

18 T. Löschner, A. Mehner, S. Grund, A. Seifert, A. Pohlers, A. Lange, G. Cox, H.-J. Hähnle and S. Spange, Angew. Chem., Int. Ed., 2012, 51, 3258-3261.

19 S. Spange, P. Kempe, A. Seifert, A. A. Auer, P. Ecorchard, H. Lang, M. Falke, M. Hietschold, A. Pohlers, W. Hoyer, G. Cox, E. Kockrick and S. Kaskel, Angew. Chem., Int. Ed., 2009, 48, 8254-8258.

20 T. Ebert, G. Cox, E. Sheremet, O. Gordan, D. R. T. Zahn, F. Simon and S. Spange, Chem. Commun., 2012, 48, 9867-9869.

21 H.-H. Huang, B. Orler and G. L. Wilkes, Polym. Bull., 1985, 14, 557-564.

22 H. H. Huang, B. Orler and G. L. Wilkes, Macromolecules, 1987, 20, $1322-1330$

23 G.-D. Kim, D.-A. Lee, J.-W. Moon, J.-D. Kim and J.-A. Park, Appl. Organomet. Chem., 1999, 13, 361-372.

24 T. Matsumoto, Advanced Materials '93: Computations, Glassy Materials, Microgravity and Non-Destructive Testing, Newnes, 1995.

25 C.-L. Chiang, C.-C. M. Ma, D.-L. Wu and H.-C. Kuan, J. Polym. Sci., Part A: Polym. Chem., 2003, 41, 905-913.

26 K. Haraguchi, Y. Usami, K. Yamamura and S. Matsumoto, Polymer, 1998, 39, 6243-6250. 